Monika Scheidler

Technical University of Dresden, Germany

\title{
Biografisches und traditionsorientiertes Erzählen als elementare Lernformen der Katechese
}

\author{
Biographical and Tradition-oriented Narration \\ as Basic Ways of Learning in Catechesis
}

\section{Abstract}

The article presents tradition-oriented as well as biographical narration as basic forms of learning in catechesis. Firstly, the Church is considered as a narrating-community and a place of learning. Secondly, the specific profile of learning processes in catechesis is elaborated. Furthermore, narration is described as an elementary form of learning and the functions of tradition-oriented as well as biography-oriented narration are pointed out. Finally, the various facets of the catechist's role as narrator are explained.

\section{Keywords}

Church as narrating community - profile of catechetical learning - telling stories as elementary method - functions and methods of biographical and tradition-oriented story-telling - role of the catechist in narrating-processes.

Der Deutsche Katecheten-Verein hat 1983 einen großen Kongress zum Thema „Erzähl mir deine Geschichte“ veranstaltet. Bei diesem Kongress haben Religionspädagogen von der Basis und aus Universitäten einander ihre persönlichen Glaubensgeschichten erzählt und gemeinsam die Erfahrung gemacht, dass das biografische Erzählen ein unverzichtbarer Teil der Katechese ist. Deshalb nehme ich im Folgenden nicht nur das traditionsorientierte Erzählen sondern auch das biografische Erzählen als zentrale Lernformen der Katechese in den Blick. Im 
ersten Schritt geht es um die Kirche als Erzählgemeinschaft und Lernort. Zweitens erläutere ich das spezifische Profil katechetischen Lernens. Drittens geht es um das Erzählen als elementare Lernform der Katechese, um die Funktionen von traditionsorientierten und biografieorientierten Varianten des Erzählens und um ihr gemeinsames Anliegen. Meine Überlegungen zum spezifischen Lernpotenzial des Erzählens beziehen sich vor allem auf die Katechese, die in christlichen Gemeinden durchgeführt wird, weil das katechetische Potenzial des Erzählens sich unter den Rahmenbedingungen des schulischen Religionsunterrichts (RU) m.E. nur begrenzt entfalten lässt. Viertens erläutere ich die verschiedenen Facetten der Rolle des Katecheten als Erzähler.

\section{Die Kirche als Erzählgemeinschaft und Lernort}

Die folgenden Überlegungen gehen davon aus, dass sich das Leben des Menschen als ein „Gewebe erzählter Geschichten“ (P. Ricoer) verstehen lässt und Menschen im Hören und Erzählen von Lebensgeschichten ihre Identität entwickeln. Eine narrative Identität, die nicht statisch, sondern dynamisch ist, haben auch Gruppen, Nationen und Kulturen. Verschiedene Gruppen haben ihre je eigenen identitätsstiftenden Geschichten, so dass jeder Einzelne in der pluralen Gesellschaft vor der Aufgabe steht, die ihm wichtigen Segmente aus diversen Lebensbereichen sinnvoll zu verknüpfen, die eigene Lebensgeschichte als Ganzes zu verstehen und anderen davon zu erzählen, damit er sich im Spiegel der anderen sehen und seine Identität weiter entwickeln kann.

Auch der biblisch-christliche Glaube bezieht sich von Anfang an auf Geschehnisse, die in der Geschichte Gottes mit dem Menschen und der Welt und in diesem Kontext mit der Erwählungsgeschichte Israels im Alten Testament erzählt werden und in der Geschichte Gottes mit Jesus Christus im Neuen Testament. Dementsprechend sind nicht nur die Glaubensbekenntnisse Israels narrativ geprägt, sondern auch die Glaubensbekenntnisse der frühen Kirche (z.B. das apostolische Glaubensbekenntnis). Das Erzählen und Erzähltbekommen von Lebensgeschichten gehört zu den ursprünglichen Vollzugsformen biblisch-christlichen Glaubens. Auch theologische Aussagen über die Bedeutung Jesu Christi oder die Rede über die Dreieinigkeit Gottes sind zusammenfassende Interpretationen einer Geschichte, die sich nur erzählend vergegenwärtigen lässt. Deshalb ist die Kirche eine „Erinnerungs- und Erzählgemeinschaft“ (J.B. Metz). Der Glaube muss zwar auch denkerisch von einer wissenschaftlich dialogfähigen Theologie 
verantwortet werden, doch die Theologie und die kirchliche Gemeinschaft leben letztlich aus dem Erzählten und dienen dem Erzählen.

In der Kirche als Institution und in der Katechese kann die Spannung zwischen der narrativen Partialität vieler einzelner Glaubensgeschichten einerseits und der systematischen Totalität einer z.B. mit dem Weltkatechismus einklagbaren „unverfälschten Ganzheit“ des Glaubens andererseits lähmend wirken und dazu führen, dass beide Bereiche auseinander fallen ${ }^{1}$. Damit entsteht eine unheilvolle Spaltung zwischen persönlicher Glaubensakzentuierung und „unverkürzter“ Katechese. An dieser Stelle lohnt es sich mit Ottmar Fuchs von der Bibel zu lernen: „Gerade die Offenbarung der Bibel, auf die sich jede systematische Theologie beruft, besteht aus nichts anderem als partiellen Glaubenserfahrungen, Glaubensakzentuierungen, Geschichten und Theologien“2. Die persönlichen Glaubensgeschichten mit ihren partiellen theologischen Deutungen sind für die Verkündigung des Glaubens der Kirche unverzichtbar und jeder einzelne hat mit seiner Geschichte ein Charisma, das in der kommunikativen Interaktion zur Quelle des Glaubens für andere wird. Dem biblischen Befund, dass von unterschiedlichen Personen unterschiedliche Glaubensgeschichten erzählt werden, entspricht die Tatsache, dass keine einzelne Person die Inhalte der christlichen Botschaft und noch weniger alle systematisch-theologischen Aspekte durch ihre persönliche Glaubenserfahrung ,abdecken“ kann. Erst die Gesamtheit der unverwechselbaren Einzelgeschichten garantiert die Ganzheit der Offenbarung. Dieses Strukturmerkmal der Bibel lädt zu einem begegnungsorientierten Verständnis des Glaubens ein, dem es nicht in erster Linie um die Ausweitung des individuellen Glaubens auf alle überlieferten Glaubensinhalte geht, sondern darum, die von der Biografie her notwendig profilierten und einseitigen Glaubenserfahrungen im Gespräch mit anderen Glaubenden zu kommunizieren und sich von den Erfahrungen anderer anregen, korrigieren und ergänzen zu lassen.

So gesehen wird der Glaube nicht in erster Linie durch das Auswendiglernen von geronnenen theologischen Formeln und Katechismussätzen tradiert, sondern durch das Austauschen persönlicher, partieller Glaubenserfahrungen. Indem Christen sich beim Erzählen ihrer persönlichen Glaubensgeschichten gegenseitig die Schrift auslegen und in der Art, wie sie miteinander sprechen und handeln, gewinnt der Glaube nicht nur lebenspraktische Plausibilität und

${ }^{1}$ Vgl. O. Fuchs, Bemerkungen und Beobachtungen zum Deutschen Katechetischen Kongress 1983, in: Deutscher Katecheten-Verein e.V. (Hrsg.), Erzähl mir deine Geschichte, Freiburg 1984, Verlag Herder, S. 152-154; ders., Spuren auf meinem Glaubensweg, in: Deutscher KatechetenVerein (Hrsg.), Dokumentation des Deutschen Katechetischen Kongresses 1983, Verlag DKV, S. 257-265.

${ }^{2}$ Ders., Bemerkungen, S. 152. 
stiftet Gemeinschaft, sondern darin erschließt sich zugleich die „Fülle“ des Glaubens der Kirche. Das Erzählen und Erzähltbekommen von persönlichen Glaubensgeschichten ist somit nicht nur für die Identitätsentwicklung des Einzelnen konstitutiv, sondern auch für den Aufbau und die Entwicklung christlicher Gemeinschaft und Kirche (Ekklesiogenesis).

Zum Verstehen der Funktionen des Erzählens im Rahmen der Weitergabe des Glaubens ist außerdem die Unterscheidung zwischen Tradition und Traditionalismus hilfreich ${ }^{3}$. H.J. Pottmeyer sieht im Verständnis von Tradition als Konstrukt bestimmter Sätze, deren Wahrheit durch die formale Autorität des Lehramtes garantiert wird, eine unzulässige Vereinseitigung des spezifisch katholischen Verständnisses von Tradition. Ein katholisch-theologisches Verständnis von Tradition im Sinne des Zweiten Vatikanischen Konzils fokussiert Pottmeyer zufolge vor aller Verbaltradition die Realtradition, in der sich die Übermittlung der Gemeinschaft mit Gott und untereinander aktuell ereignet. Für die Spannung zwischen Tradition und Veränderung in der Kirche bedeutet dies, dass die Glaubensgemeinschaft das „Wachstum der Treue“ gegenüber ihrer Berufung je neu auszubalancieren hat. Pottmeyer sagt: „Wenn die Tradition vornehmlich als Verbaltradition in Form von Lehrsätzen verstanden wird, dann erscheinen diejenigen, die diese Sätze bilden und deren Wahrheit garantieren [die Amtsträger, M.S.], als die eigentlichen menschlichen Subjekte der Tradition. Wird aber gesehen, dass die Verbaltradition nur dann zu ihrer Bestimmung findet, wenn sie in den Herzen, in der Einsicht und Praxis der Gläubigen zur Realtradition wird, zur communio mit Gott und untereinander, dann sind alle wahren Rezipienten und Tradenten [also auch die gläubigen Laien, M.S.] ministeriale Subjekte der Tradition" ${ }^{\text {“4 }}$ Versteht man Tradition dementsprechend als Geschehen, das von vielen Gläubigen bzw. der ganzen Glaubensgemeinschaft getragen wird, kommt zum einen die große Bedeutung der Katechese als Ort der Tradierung bzw. als Lernort der christlichen Botschaft in den Blick und zum anderen die Bedeutung von haupt- und ehrenamtlichen Laien, die als Katecheten oder als Teilnehmende an der Katechese selber Subjekte der Tradition sind. So gesehen trägt in der Katechese speziell das biographische Erzählen eigener Glaubensgeschichten, die sich bezeugend mit biblisch-christlichen Quellen verknüpfen, zur Konstituierung der Kirche und ihrer Zukunftsfähigkeit bei.

\footnotetext{
${ }^{3}$ Vgl. H.J. Pottmeyer, Die Suche nach der verbindlichen Tradition und die traditionalistische Versuchung der Kirche, in: D. Wiederkehr (Hrsg.), Wie geschieht Tradition? Überlieferung im Lebensprozess der Kirche (Quaestiones Disputatae 133), Freiburg 1991, Verlag Herder, S. 89-110.
}

${ }^{4}$ Ebd., S. 105. 


\section{Das Profil katechetischen Lernens}

Auf der Basis von Fachliteratur und einschlägigen kirchlichen Dokumenten verstehe ich unter Katechese im engen Sinn die im persönlichen Kontakt und gemeinschaftlich erfolgende zielgerichtete und strukturierte Auseinandersetzung mit dem Zuspruch und Anspruch des christlichen Glaubens. Als solche ist die Katechese ein Grundvollzug des mit der Taufe in Gang gesetzten (oder bei Taufbewerbern in Gang zu setzenden) Lebens in der Gottesbeziehung und der Gemeinschaft der Kirche. Der wichtigste Lernort der Katechese im engen Sinn ist die christliche Gemeinde.

Der Religionsunterricht in der Schule kann zwar durchaus zur Erstbegegnung von jungen Menschen mit der Frohen Botschaft beitragen und die Evangelisierung getaufter Schüler unterstützen, aber der zentrale Ort des Lebens und Feierns christlichen Glaubens kann die Schule m.E. nicht sein, weil Lehrer und Schüler im RU nur in begrenzten Zeitfenstern miteinander lernen und leben. Um nicht nur etwas über den christlichen Glauben zu lernen, sondern auch aus dem Glauben leben zu lernen, brauchen Menschen mehr Kontaktzeit mit lebendigen Christen, in der sie auch das praktische Leben von Christen kennen lernen und sich mit ihrem Lebens- und Wortzeugnis aktiv auseinandersetzen, so dass sie zur Entscheidung kommen können, auch selbst als Christ zu leben und die eigenen Lebenserfahrungen im Licht des Glaubens zu deuten. Dies ist im schulischen Unterricht nur begrenzt möglich, sondern eher im Kontext von Familie, Gemeinde und anderen freundschaftlichen Netzwerken realisierbar, wenn Menschen etwas von ihrem Glauben im Zeugnis des Lebens und Wortes miteinander teilen, sich gegenseitig im Glauben bestärken und korrigieren ${ }^{5}$.

Das zentrale Ziel der Katechese im engen Sinn besteht darin, zur Entfaltung der Taufberufung von Menschen beizutragen, bezieht sich auf die Grundaufgaben von Christen und Kirche in der Welt.

${ }^{5}$ In der Katechese im engen Sinn hat die Kirche in erster Linie eine innerkirchliche Bildungsaufgabe zu erfüllen und zur Entfaltung der Taufberufung beizutragen, während sie im schulischen RU, an dem in vielen Ländern auch interessierte nichtkatholische Schüler teilnehmen, einen gesellschaftlichen Bildungsauftrag zu erfüllen hat. Im schulischen RU haben die Religionsgemeinschaften vor allem einen Dienst an der religiösen Bildung junger Menschen im Rahmen der schulischen Allgemeinbildung zu leisten - mit dem Ziel, dass diese jungen Menschen als mündige Bürger ihres Landes so mit der rechtlich garantierten Religionsfreiheit umgehen lernen, dass sie sich mit eigenen Gründen für oder gegen die Zugehörigkeit zur Kirche oder einer anderen Religionsgemeinschaft entscheiden können. Weil die Lerngruppen [Klassen] an öffentlichen Schulen, in denen Kath. RU erteilt wird, in der Regel heterogener sind als die Katechesegruppen, die in kath. Gemeinden z.B. zur Vorbereitung auf ein Sakrament zusammen kommen, muss der schulische RU grundsätzlich ergebnisoffener sein als die Katechese in der Gemeinde. 


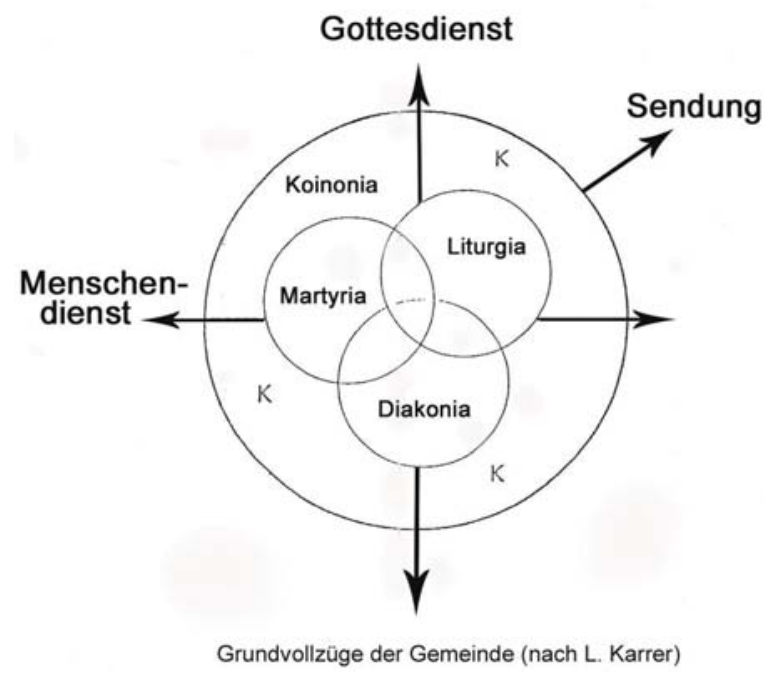

Die Grundvollzüge und Aufgaben der Gemeinde ${ }^{6}$ bzw. Kirche korrespondieren mit den Zielbereichen der Katechese im engen Sinn, in der Menschen lernen,

- wie das Leben des Einzelnen in der Koinonia mit Gott und in der kirchlichen Gemeinschaft gelingen kann,

- wie Koinonia erfahren und aufgebaut wird: im konkreten Dienst an schwächeren Menschen (Diakonie), im Feiern des Glaubens (Liturgie) und im Bezeugen des Glaubens in Wort und Tat (Martyria - nicht zuletzt durch das Erzählen von Glaubensgeschichten),

- welche Art von Auftrag (Sendung) die Koinonia der Christen und der Einzelne als Vertreter der kirchlichen Gemeinschaft im gesellschaftlichen Umfeld hat und wie sich der jeweilige Auftrag realisieren lässt.

Zentrale Inhalte der Katechese im engen Sinn sind die elementaren Strukturen des christlichen Bekenntnisses, der Feier der Sakramente, des Betens von Christen und der Gebote als Richtschnur christlicher Lebenspraxis ${ }^{7}$. Durch die Erschließung der Kernstrukturen christlichen Glaubens und Lebens sollen

${ }^{6}$ Vgl. L. Karrer, Grundvollzüge christlicher Praxis, in: H. Haslinger u.a. (Hrsg.), Handbuch Praktische Theologie, Bd. 2, Mainz 2000, Verlag Grunewald, S. 379-395.

${ }^{7}$ Der schulische RU hat zum einen ein größeres inhaltliches Feld abzudecken als die Katechese im engen Sinn, weil der RU auch Wissen über andere Konfessionen und Religionen vermitteln und die Schüler zum Gespräch mit Christen anderer Kirchen, mit Angehörigen anderer Religionsgemeinschaften und mit Menschen nichtreligiöser Weltanschauungen befähigen soll. Zum anderen können am RU in der Regel auch nichtkatholische Schüler teilnehmen, so dass die Lerngruppen heterogener sind als in der Katechese im engen Sinn und der RU deshalb faktisch auch nur begrenzt zur Entfaltung der Taufberufung der katholischen Schüler beitragen kann. 
diejenigen, die an der Katechese teilnehmen, befähigt werden entsprechend ihrer Taufberufung als Christen zu glauben, zu leben und zu handeln. Für die Katechese ist somit eine Doppelbewegung zwischen den Inhalten einerseits und den Lernenden andererseits erforderlich.

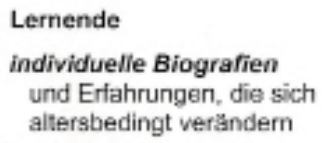

\section{Doppelbewegung}

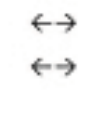

Korrelation

\author{
Sache \\ Inhalte der biblisch-christichen \\ Tradition, die auf Erfahrungen \\ von Glaubenden früherer Gene- \\ rationen basieren und bei Men- \\ schen heute Fragen, Zweifel oder \\ Gewissheit provozieren
}

Dafür, dass diese Doppelbewegung (fachsprachlich: Korrelation) gelingt, ist das Erzählen eine besonders wichtige Lernform. Bezogen auf die Seite der Inhalte sei daran erinnert, dass die Kirche wesensgemäß eine Erzählgemeinschaft ist und letztlich auch theologische Reflexion immer auf Erzähltem basiert und dem Erzählen dient. Zugleich ist die Kirche als lebendige Erzählgemeinschaft, die in der Gegenwart Verknüpfungen zur biblisch-christlichen Tradition entwickelt, aber auch herausgefordert nicht dem Traditionalismus zu verfallen, sondern als Erinnerungsgemeinschaft, aktiv an ihrer Zukunftsfähigkeit zu arbeiten. Auf der Seite der Lernenden ist es wichtig, sich in kleinen Gruppen von den individuellen Lebens- und Glaubenserfahrungen zu erzählen. Die dadurch entstehende Gemeinschaft ist eine Erzählgemeinschaft, die sich als kirchliche Gemeinschaft immer auch an das von Christen vor uns Erzählte zurück zu binden hat und zugleich anknüpfend an diese Tradition zeitgemäße neue Geschichten aus dem gegenwärtigen Erfahrungskontext erzählt und heute Erlebtes christlich deutet.

Eine für das Erzählen aufschlussreiche Differenzierung dieser Doppelbewegung zwischen individuellen Biografien und Inhalten der biblischen Tradition kommt aus lernpsychologischer und didaktischer Perspektive in den Blick, wenn man von drei Seiten des Lehrens und Lernens ausgeht: von einem didaktischen Dreieck (Lehrende-Lernende-Sache), vom Dreieck der Themenzentrierten Interaktion (Ich-Wir-Thema) oder vom sog. Lerndreieck nach Weinert und Hasselhorn ${ }^{8}$ :

${ }^{8}$ Vgl. M. Hasselhorn, A. Gold, Pädagogische Psychologie. Erfolgreiches Lehren und Lernen, Stuttgart 2006, Verlag Kohlhammer. 


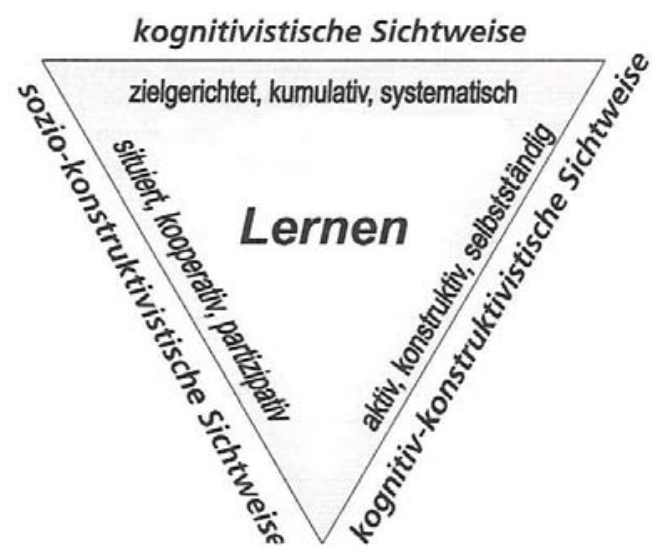

Drei Seiten des Lernens (nach F.E. Weinert)

Die drei Sichtweisen des Lernens, die dieses Dreieck synthetisiert, spiegeln die aktuellen Lehr-Lerntheorien:

- kognitivistische Sichtweise in der verhaltensorientierten Tradition (Skinner, Gagné) und der kognitiv-rationalistischen Tradition (Gagné, Ausubel)

- kognitiv-konstruktivistische Sichtweise in der Tradition von Piaget, Bruner, Aebli

- sozio-konstruktivistische Sichtweise in der Tradition von Wygotski, Brown, Rogoff.

Aus kognitivistischer Sicht wird Lernen als Wissenerwerb verstanden und geschieht durch Information, Assoziation, Wiederholen und Reagieren auf Verstärkung bzw. Bestrafung sowie die daraus resultierende Informationsverarbeitung. Lehren wird als Instruktion verstanden und geschieht primär durch die Vermittlung strukturierter Wissenspakete (Katechismuswissen). Der Lehrende versteht sich in erster Linie als Informationsvermittler, der „im Besitz" des Wissens bzw. der curricular definierten Inhalte ist. Er behandelt die Lernenden als passive Informationsempfänger, die Wissen rezipieren sollen und beispielsweise erst dann zur Erstkommunion gehen dürfen, wenn sie drei Grundgebete und die Wandlungsworte auswendig können. Diese Sichtweise des Lehrens und Lernens ist m. E. beim katechetischen Lernen im engen Sinn weniger wichtig. Mit anderen Worten: Wer katechetisches Lernen ausschließlich aus kognitivistischer Sicht versteht und realisiert, kann nicht zum Kern dessen kommen, worauf Kirche und Theologie sowie ihre Zukunftsfähigkeit gründen: zum Erzählen der Erfahrungen von Glaubenden vor uns in der Geschichte Israels und der Geschichte Jesu Christi einerseits und zum Erzählen-lassen der 
Erfahrungen heutiger Christen mit ihren Fragen und Zweifeln andererseits, die ihren Glauben im jeweiligen Umfeld aktualisieren.

Aus kognitiv-konstruktivistiche Sicht wird Lernen als Wissenskonstruktion des Einzelnen durch Information und eigene Entdeckung verstanden. Lehren wird dementsprechend als Reaktion auf das Lernen von Einzelnen bzw. als Endecken-lassen realisiert. Der Lehrende versteht sich dabei nicht in erster Linie als Informationsvermittler, sondern als Impulsgeber und Lernbegleiter. In den Lernenden sieht er eigenständige, aktive Subjekte, die Lerninhalte in subjektiver Weise verstehen, mit ihrem Vorwissen verknüpfen und ihr Wissen ausbauen. Der Lernende wird in dieser Konzeption also nicht als passiver Konsument verstanden, der Informationen von außen identisch abbildet und wiedergibt, sondern er wird als aktiver Konstrukteur von Bedeutungen gesehen, der aus neuen Informationen auf der Basis eigener Vorerfahrungen Sinn generiert und in seine bestehenden Wissensstrukturen integriert. Aus konstruktivistischer Sicht ist das Lernen somit nur teilweise von außen steuerbar und die Lernergebnisse sind nur bedingt vorhersehbar. Diese Sichtweise entspricht den Anliegen biographischen Lernens und ist m.E. für die Katechese im engen Sinn angemessener als die zuvor beschriebene ausschließlich kognitivistische Sicht, weil die individuellen Erfahrungen der Lernenden und das Erzählen davon für das katechetische Lernen konstitutiv sind.

Aus sozio-konstruktivistischer Sicht wird Lernen als Wissenskonstruktion und mit anderen geteilte Kognition verstanden, so dass Lehren primär durch das gemeinsame Aushandeln (Konstruieren) von Bedeutungen und das Bereitstellen von Lernumgebungen bzw. Problemsituationen ${ }^{9}$ realisiert wird. In dieser Sichtweise geht man davon aus, dass Wissen niemals unabhängig von dem sozialen und kulturellen Kontext erworben wird, in dem es erlernt wird. Die Lehrperson hat deshalb in erster Linie dafür zu sorgen, dass das Lernen in einem geeigneten Kontext geschieht, in dem die Bedeutung des jeweiligen Lerninhalts erfahren und schließlich - über mehrere Anwendungssituationen hinweg - abstrahiert werden kann. Gleichzeitig wird Lernen als sozialer Vorgang durch Interaktionen verstanden und derAufbau von Wissenstrukturen als soziale Konstruktionsleistung der jeweiligen Lerngruppe betrachtet. Die Interaktionsfähigkeit der Lehrperson mit den Lernenden und der Lernenden untereinander sind dafür zentrale Voraussetzungen. Auch aus sozio-konstruktivistischer Sicht ist katechetisches Lernen also viel mehr als das Auswendiglernen von Katechismuswissen, weil Lernen in dieser Perspektive gleichbedeutend mit der Fähigkeit ist, an den

${ }^{9}$ Eine authentische Lernumgebung und Problemsituation wird z.B. geschaffen, wenn in die Firmkatechese ein Diakonieprojekt integriert ist, an dem die Jugendlichen sich beteiligen. 
Praktiken einer Gemeinschaft teilzuhaben. Bei solchen Lernprozessen wird somit nicht nur Glaubenswissen angeeignet, sondern auch eine in der jeweiligen Glaubensgemeinschaft geteilte und durch den Katecheten vermittelte Haltung, die die Bedeutung dieses Wissens kommuniziert und nicht zuletzt das Menschenbild sowie die Orientierungsmuster, die den Praktiken der Gemeinschaft zu Grunde liegen. Die sozio-konstruktivistische Sichtweise des Lernens korrespondiert m.E. am besten mit dem zentralen katechetischen Anliegen, weil es in der Katechese (zur Entfaltung der Taufberufung in der persönlichen Gottesbeziehung und im Mit-Leben mit der Kirche) vor allem einer persönlichen und gemeinschaftlichen Auseinandersetzung mit dem Zuspruch und Anspruch des christlichen Glaubens bedarf. Solche Auseinandersetzung mit dem christlichen Glauben sollte m.E. in der Katechese im engen Sinn sogar Vorrang haben vor der systematischen Unterweisung, weil es für die Kirche als Erzählgemeinschaft konstitutiv ist, den Erzählungen vom Leben heute glaubender Menschen zu folgen - in lebendiger Treue zu den biblisch-christlichen Quellen.

Weil die Lernergebnisse des schulischen RU im Unterschied zu Lernergebnissen der Katechese im engen Sinn in der Regel mit Noten zu bewerten sind und der RU (wie andere Schulfächer) einem relativ festen Lehrplan zu folgen hat, muss der Schwerpunkt des schulischen RU deutlicher als bei der Katechese in der Gemeinde auf dem ergebnisorienierten, kumulativen und systematischen Lernen liegen. In der Katechese im engen Sinn gibt es m.E. viel mehr Freiräume für aktives, konstruktives und selbstständiges Lernen der Einzelnen sowie für situierte und partizipative Lernprozesse, die für die Weitergabe des Glaubens unabdingbar sind. In den folgenden Überlegungen zum Lernpotenzial des Erzählens beziehe ich mich auf die Katechese im engen Sinn, weil das große Lernpotenzial des Erzählens im schulischen RU wegen seiner anderen Rahmenbedingungen und seines weiteren Spektrums von Zielen und Inhalten nur begrenzt zur Entfaltung kommen kann.

\section{Erzählen als elementare Methode der Katechese}

Das Erzählen ist zwar eine traditionelle und angesichts vielfältiger neuer Medien und Methoden leicht in Vergessenheit geratende Lernform. Wenn das Erzählen aber ernst genommen wird und beispielsweise als dialogisches Erzählen die traditionelle Form des Frontalunterrichts bewusst durchbricht, kann es in der Katechese durchaus zum doppelpoligen Erschließungsprozess zwischen Lernenden und Lerngegenstand beitragen. Nach F. Schweitzer lassen sich nur 
solche Methoden als elementare Lernformen der Katechese bezeichnen, die diesem doppelpoligen Erschließungsprozess gerecht werden ${ }^{10}$.

Beim Erzählen handelt es sich um eine elementare Lernform der Katechese, insofern...

1. Erzählungen den Lebens- und Handlungsbezug eines Themas anschaulich machen und in religiösen Lernprozessen dazu beitragen, dass das Erzählte Bedeutung für das Leben der Lernenden gewinnen und bei ihnen etwas bewirken kann.

2. Erzählungen in Situationen existentieller Auseinandersetzung mit weltanschaulich-religiösen Wahrheits-und Gewissheitsansprüchen wurzeln, so dass das Erzählte innere Bilder entstehen lässt, mit denen Erzähler und Zuhörer eigene Gefühle und Wertungen assoziieren, und die Identitätsentwicklung angeregt wird.

3. das Medium der Erzählung ein Angebot ist, das die Hörer/Leser grundsätzlich frei lässt und sie zur Perspektivenübernahme einlädt, so dass diejenigen, die sich darauf einlassen, befähigt werden, sich selber mit den Augen des Erzählten und vom Erzählten her zu sehen,

4. das Erzählen ein intersubjektives Geschehen ist, in dem die Geschichte eines Subjekts (oder mehrerer) mitgeteilt wird, und somit auch der Erzähler (Lehrer/ Katechet) selbst herausgefordert ist, die Zuhörer (Lernenden/Katechumenen) als freie Subjekte mit eigenen Wahrnehmungen ernst zu nehmen und sich auf manchmal überraschende kognitive Konflikte in der Auseinandersetzung mit dem Erzählten einzulassen,

5. das Erzählen entsprechend seiner kommunikativen Intention besonders beim freien, kreativen Erzählen eigener Erlebnisse oder Phantasiegeschichten die aktive Auseinandersetzung der Lernenden mit existentiellen Fragen anregt, so dass es in der Katechese nicht nur dazu beitragen kann, das Selbstverständnis der Lernenden zu erweitern sondern auch zur kritisch-produktiven Tradierung biblisch-christlicher Erfahrungen anregt.

Das Erzählen knüpft in der Katechese entweder bei den lernenden Personen und ihren Erfahrungen an oder bei tradierten Erzählungen der Bibel und der Geschichte des Christentums. Weil das biografiebezogene Erzählen der Lernenden ein anderes Potenzial enthält als das traditionsorientierte Erzählen, werde ich diese

${ }_{10}$ Vgl. F. Schweitzer, Elementarisierung - ein religionsdidaktischer Ansatz. Einführende Darstellung, in: ders., Elementarisierung im Religionsunterricht, Neukirchen-Vluyn 2003, Verlag Neukirchener, S. 24-26; ders., Elementarisierung nur der Inhalte - oder elementare Formen des Lernens? in: ebd., S. 190-193. 
beiden Ansätze zunächst differenziert beschreiben, bevor ich ihr gemeinsames Anliegen und die Herausforderung ihrer Verknüpfung in den Blick nehme.

\subsection{Biografisches Erzählen in der Katechese}

Demjenigen, der von sich erzählt, steht ein eigenes Erlebnis vor Augen, das ihn noch beschäftigt. F.W. Niehl erläutert: „Beim Erzählen vergegenwärtigt er sich dieses Erlebnis wieder; er erlebt noch einmal, was ihn ... bewegt hat. Die inneren Bilder, die dabei entstehen, seine Gefühle und seine Wertungen formen ... die Art, wie er davon erzählt: Wortwahl, Satzbau, die sprachliche Gestaltung insgesamt und nicht zuletzt Mimik und Gestik "“11. Solches Erzählen ist eine nicht ersetzbare Form menschlicher Sprache mit folgenden Funktionen:

1. Vergegenwärtigung, Artikulation und Deutung des in einer bestimmten Situation vom Erzähler selbst Erlebten

2. Kommunikation über das Erlebte zwischen Erzähler und Zuhörern

3. Konstitution von Wirklichkeit, Wertungen und Orientierungsmustern für das Handeln im Medium der Erzählung

4. Entwicklung individueller und sozialer Identität in der Auseinandersetzung mit den Wertungen und Orientierungsmustern im Erzählten

5. Anregung bzw. Öffnung neuer Erfahrungen mit dem Erzählten, das von den Zuhörern in Freiheit abgelehnt, übernommen oder modifiziert werden kann zur Verarbeitung und Deutung eigener Erlebisse.

Beim biografischen Erzählen erinnert ein Ich-Erzähler sich nicht nur an ein Erlebnis, sondern er greift gezielt mehrere Erlebnisse seiner Lebensgeschichte auf, die ihn heute noch bewegen, die seine Gefühle, Wertungen und sein Selbstverständnis prägen. Er verbindet die ihm wichtigen Erlebnisse mit einem „roten Faden“, indem er wie beim Aufziehen einer Kette einige sorgfältig ausgewählte Perlen mit eigenen Wertungen und Deutungen auf eine Schnur nimmt. Beim biografischen Erzählen konkretisieren die Funktionen des Erzählens sich dahingehend, dass der Erzähler sein Selbstverständnis im Spiegel der Zuhörer klärt und dabei seine Identität im aktuellen sozialen und kulturellen Rahmen der Erzählgemeinschaft profiliert. Das Lernpotenzial biografischen Erzählens liegt somit in der Vergewisserung des Erzählers zur Frage: Wer bin ich heute mit meiner Lebensgeschichte im Kreis meiner Zuhörer?

Für die Katechese sind kleine Ich-Erzählungen und längere biografische Erzählungen der Katecheten ebenso wie der Teilnehmenden unverzichtbar,

${ }^{11}$ F.W. Niehl, Erzählen, in: L. Rendle (Hrsg.), Ganzheitliche Methoden im Religionsunterricht, München 2007, Verlag Kösel, S. 165. 
weil Erzählern und Zuhörern damit ein Raum eröffnet wird, alltägliche Lebenssituationen als existentielle Situationen wahrzunehmen, sich mit anderen darüber auszutauschen, ihre Erlebnisse im Kontext der Katechesegruppe in tastenden Erzählversuchen auch im Licht des Glaubens zu sehen und etwas von den ureigenen Gottes- und Glaubenserfahrungen mitzuteilen. Biografische Erzählungen, in denen ein Erzähler Erlebisse der eigenen Lebensgeschichte mit Erfahrungen von anderen Glaubenden (z.B. früherer Generationen) verknüpft, sind elementare Lernformen religiöser Bildung. Solche Verknüpfungen können beim biografischen Erzählen aber erst entstehen, wenn der Erzähler motiviert ist, eigene Erlebnisse bewusst mit Bezug auf die Glaubensgemeinschaft und ihre Tradition zu verstehen, und er dies auch gegenüber seinen Zuhörern deutlich macht (bezeugt). Das bezeugende Erzählen des Katecheten oder eines Teilnehmenden kann auch andere Teilnehmende anregen, beim Erzählen ihrer Geschichten Ähnliches zu probieren.

\subsection{Traditionsorientiertes Erzählen in der Katechese}

Kulturell tradierte Erzählungen dienen als Verständigungstexte, die sich vor allem dann ins zwischenmenschliche Gespräch einmischen, wenn es um Ziele und Werte menschlichen Handelns geht. Sie provozieren emotionale Reaktionen, bieten Orientierungsmuster an und laden dazu ein, eigene Verhaltensmuster zu überprüfen und nötigenfalls zu ändern. Durch traditionsorientiertes Erzählen werden unsere Wahrnehmungs- und Deutungsmuster erweitert und wir erhalten Impulse zur Weiterentwicklung unserer individuellen und sozialen Identität. Das traditionsorientierte Erzählen hat trotz seines Ansatzes bei überlieferten Erzählungen aus anderen sozialen und kulturellen Kontexten ähnliche Funktionen wie das biografische Erzählen ${ }^{12}$. Die Geschichten, die uns aus der Vergangenheit von Menschen vor uns überliefert werden, unterscheiden sich von biografischen Erzählungen heute lebender Menschen vor allem durch ihre größere Distanz zu den Rezipienten. Wenn wir als typische katechetische Situation, in der IchErzählungen und biografische Erzählungen vorkommen, einmal annehmen, dass lebendige Menschen aus dem allen bekannten sozialen Umfeld etwas von ihrer Lebens- und Glaubensgeschichte erzählen, haben die Zuhörer in der Regel eine größere Nähe zum Erzähler und dem Erzählten als bei der Konfrontation mit

\footnotetext{
${ }^{12}$ Funktionen des traditionsorientierten Erzählens sind: 1. Vergegenwärtigung, Artikulation und Deutung der existentiellen Situation, die in der Erzählung überliefert ist, 2. Kommunikation über das, was von der Erzählung überliefert ist, 3. Konstitution von Wirklichkeit und Orientierungsmustern im Medium der Erzählung, 4. Entwicklung individueller und sozialer Identität in Auseinandersetzung mit der überlieferten Erzählung und 5. Anregung neuer Erfahrungen, die an Erfahrungen der Überlieferung anknüpfen.
} 
überlieferten Erzählungen. Beim biografischen Erzählen brauchen die Zuhörer also nicht erst einen ,garstigen Graben“ in eine andere soziale und kulturelle Welt und Zeit zu überwinden wie beim traditionsorientierten Erzählen.

Elementares Lernen kann aber durchaus auch beim traditionsorientierten Erzählen gelingen, wenn die Lernenden sich so in den Bann der Erzählung ziehen lassen und sich auf ihre Abläufe einlassen, dass sie sich herausgefordert sehen, selbst Stellung zum erzählten Geschehen zu beziehen und sich aktiv mit dem Erzählten auseinandersetzen. Ein plastisches Beispiel hierfür ist eine beobachtete Sequenz aus einer Religionsstunde mit 11jährigen Schülern über das Gottesurteil auf dem Karmel (1Kön 18). Die Schüler ließen sich ganz in den Bann der Erzählung ziehen, in der Elia zunächst das Baal verehrende Volk auffordert, einen Opferaltar mit einem Stier herzurichten und Baal um Feuer anzurufen, während Elia einen Opferaltar baute und den Gott Abrahams um Feuer anrief. Interessanterweise sahen die Schüler dieser 6. Klasse den zentralen Konflikt der Erzählung gar nicht in der Frage, wer der wahre Gott ist bzw. an welchen Gott wir unser Herz hängen wollen und warum, sondern vor allem in der Frage, warum Elia die Baalspropheten töten lässt (V. 40), obwohl er doch so stark an Gott glaubt. Die Schüler haben sich ganz auf die spannenden Abläufe der Erzählung eingelassen. Deshalb sahen sie sich herausgefordert, selbst Stellung zu beziehen zum erzählten Geschehen. Weil die Kinder diese Geschichte vom Gottesurteil am Karmel nicht ohne den Widerstand ihrer Zweifel, Anfechtung und Ablehnung wahrgenommen haben, konnten sie mit dem vom biblischen Erzähler Erzählten in ein tiefes Gespräch kommen.

D. Reiher identifiziert fünf Konzeptionen des Erzählens biblischer Geschichten ${ }^{13}$, die für die Differenzierung traditionsorientierter Erzählkonzeptionen insgesamt geeignet sind:

1. Erzählen als Darbietung der geschichtlichen Wahrheit vom Handeln Gottes, wobei der Bibeltext dazu tendiert „museal“ zu wirken und offenes Fragen zu verhindern.

2. Erzählen als exemplarische Darbietung einer Lehre: Die Erzählung soll christliche Wahrheiten verdeutlichen, interpretieren, entfalten bzw. lehren, was ebenfalls offene Fragen eher verhindert als fördert.

3. Erzählen als auslegendes Erzählen: Die Darbietung erfolgt unter Einbezug exegetischer Erkenntnisse und theologischer Interpretation, so dass Bezüge

${ }^{13}$ Vgl. D. Reiher, Praxis Christenlehre. Biblisches Erzählen in der Säkularität, in: G. Doyé, H. Kessler (Hrsg.), Konfessionslos und religiös. Gemeindepädagogische Perspektiven, Leipzig 2002, Verlag Leipziger, S. 145-158. 
zu den lebensweltlichen Erfahrungen der Hörer entstehen und ihre Neugier geweckt wird.

4. Dialogisches Erzählen: Indem der Erzähler auf das Befremdliche, Überraschende der biblischen Geschichte verweist bzw. die Differenz zu den Hörenden aufzeigt, unterstreicht er die Pointe des biblischen Textes und lädt die Zuhörer ein, sich aktiv mit der Kernaussage auseinanderzusetzen.

5. Erzählen als Umgang mit Erfahrungen: Der Erzähler stellt eine möglichst enge Korrelation her zwischen biblischem Text und den Alltagserfahrungen damals und heute, indem er „Leerstellen“ des Textes und Andeutungen von Problemen und Konflikten entfaltet, die Zuhörer in die Fragen, Probleme und Konflikte verwickelt und ihre Positionierung provoziert.

Die letzten drei Erzählkonzeptionen haben das Anliegen, den „garstigen Graben“ zwischen der Situation bzw. dem Erfahrungskontext, in dem die überlieferten Glaubensinhalte wurzeln, und der Lebenssituation der Lernenden zu überwinden. Insbesondere die 4. und 5. Konzeption, das dialogische Erzählen sowie das Erzählen als Umgang mit überlieferten und heutigen Erfahrungen, enthalten angesichts der überall zunehmenden Säkularisierungsprozesse (mit ihren Erscheinungen der Entkirchlichung, des Traditionsabbruchs und der Konfessionslosigkeit) große Lernpotenziale, weil auch heutige Menschen Kinder, Jugendliche und Erwachsene - ihre „Theologie“ bzw. ihre eigene Sicht vom Leben und Glauben haben, die sie ihrerseits erzählen möchten und bei diesen Erzählkonzeptionen auch ins Gespräch bringen sollen. Erzählungen der Bibel und der Christentumsgeschichte, die keine Lehrsätze mitteilen, sondern erzählen, wie sich Gott in den Erfahrungen von Glaubenden vor uns ereignet hat, können Menschen heute Impulse geben, auch ihre eigenen Gottes- und Glaubensgeschichten zu erzählen und mit den tradierten Geschichten ins Gespräch bringen. Dazu muss den Lernenden durch die Realisierung einer geeigneten Erzählkonzeption allerdings auch Raum gegeben werden. Wer heute biblische Geschichten erzählt, sollte seine Erzählung deshalb so gestalten, dass die Zuhörer ermutigt werden,

- sich ihre eigenen Gedanken zum Erzählten zu machen,

- ihre eigenen Sichtweisen zur Geschichte ins Gespräch zu bringen,

- sich auch spekulativ zu den in der Erzählung aufgeworfenen Fragen zu äußern,

- von eigenen Erlebnissen zu erzählen, die sie mit der Geschichte assoziieren. 
Damit - beim dialogischen Erzählen und beim Erzählen als Umgang mit überlieferten und heutigen Erfahrungen - tatsächlich der „garstige Graben“ zwischen der Lebenssituation der Lernenden und der überlieferten Erzählung überbrückt wird, sollte der Katechet,

- die Teilnehmenden einladen, wirklich ihre eigenen Gedanken und Sichtweisen zum Erzählten zu äußern, und sich auf das damit eröffnete Gespräch einlassen,

- die Übertragung der überlieferten Geschichte auf heutige Lebenssituationen und Konflikte nicht (vorschnell) vorgeben,

- sein eigenes Verständnis der Erzählung und sein eventuell damit verbundenes persönliches Zeugnis (so wichtig dies sein kann) erst in einem späteren Teil der Katechese mitteilen.

Zwischen einer Katechese, die bei den Lernenden und beim biografischen Erzählen ansetzt, und einer Katechese, die beim Erzählen überlieferter Geschichten ansetzt, öffnet sich eine Spannung, die es in der Praxis auszubalancieren gilt.

\subsection{Das gemeinsame Anliegen von biografischem und traditionsorientiertem Erzählen}

Der immer wieder erhobenen Forderung, die Katechese habe den Glauben der Kirche vollständig weiterzugeben, ist aus katechesedidaktischer Sicht entgegen zu halten: Die Katechese hat zwar auf den Glauben der Kirche insgesamt und seine in der Theologie reflektierte Botschaft zurückzugreifen, aber ihre zentrale Aufgabe besteht darin, den Kern der christlichen Botschaft für Menschen heute so zu erschließen, dass er sie in ihren Freuden und Hoffnungen, in ihren Ängsten und Sehnsüchten betrifft und ihnen Wege lebendigen Lebens eröffnet ${ }^{14}$. Dazu ist von Seiten des Katecheten die Reflexion der überlieferten Inhalte und Erzählungen im Blick auf die Menschen erforderlich, die hier und heute an der Katechese teilnehmen - wie z.B. die Jugendlichen, die sich auf die Firmung im nächsten Jahr vorbereiten. Der Reichtum der biblisch-christlichen Überlieferung ist in seiner Fülle zwar ein großer Schatz, aber er kann Lernende auch verwirren und überfordern. Kein Einzelner kann sich mit allem, was zu diesem Schatz gehört, vollständig vertraut machen. Weil die Überlieferung aber entscheidend eine Botschaft für die Armen und Kleinen ist, muss sie im Kern etwas Einfaches sein - kurz gesagt: das Angebot der Gnade Gottes bzw. der Anruf, durch den der Gott und Vater Jesu Christi die Geschichte des Einzelnen und der Menschheit in

${ }^{14}$ Vgl. Zum Folgenden D. Emeis, K.H. Schmitt, Handbuch der Gemeindekatechese, Freiburg 1986, Verlag Herder, S. 88ff. 
eine lebendige Zukunft führen möchte. So gesehen kommt es in der Katechese vor allem darauf an, den grundlegend einfachen Kern der christlichen Botschaft zu erschließen und das, was damit elementar verbunden ist.

Bei der Auswahl überlieferter Erzählungen für die Katechese ist deshalb zu klären:

- Welches für den christlichen Glauben grundlegende Zeugnis kann den Teilnehmenden in ihrer aktuellen Lebenssituation durch die Erzählung beispielhaft erschlossen werden?

- Welche für den christlichen Glauben grundlegenden Einstellungen, Haltungen, Orientierungsmuster und lebenspraktischen Entscheidungen können die Teilnehmenden in ihrer aktuellen Lebenssituation durch die Auseinandersetzung mit der Erzählung entwickeln und einüben?

Wenn man in der Katechese umgekehrt bei der Lebenssituation der konkreten Teilnehmenden ansetzt, ist von diesen Situationen (von den darin enthaltenen Konflikten, Problemen und Potenzialen) her zu fragen, was die Teilnehmenden zur Bewältigung dieser Situationen brauchen. Dabei werden auch überlieferte Glaubensinhalte und Erzählungen wichtig und hilfreich, aber sie kommen von einer anderen Fragestellung her in den katechetischen Prozess. Am Anfang dieses Ansatzes steht die Analyse der Lebenssituation der Teilnehmenden, mit ihren Konflikten, Problemen und Entwicklungspotenzialen, und erst dann wird gefragt:

- Welche überlieferten Glaubensinhalte und Erzählungen haben Bedeutung für die Annahme und Bewältigung dieser Lebenssituation durch die Teilnehmenden?

Bei diesem Vorgehen stehen die zu vermittelnden Glaubensinhalte und die zu erzählenden Geschichten nicht von vornherein fest, sondern von der Analyse der Lebenssituation her muss der Katechet die bedeutsamen Inhalte und Geschichten ermitteln. Das setzt natürlich voraus, dass er selber mit den Kernelementen der Glaubenstradition gut vertraut ist, um das, was davon für die Bewältigung dieser Lebenssituation wichtig ist, überhaupt einbringen zu können. Sicherlich muss der Katechet aber auch beim Ansatz von Überlieferung her mit der Lebenssituation der konkreten Lernenden vertraut sein, um eine Erzählkonzeption zu realisieren, die den „garstigen Graben“ zwischen vergangenen und heutigen Situationen überbrückt.

Die Stärken eines von heutigen Biografien ausgehenden Erzählens liegen in der Nähe solcher Erzählungen zum eigenen Erleben und darin, 
dass bei solchen Gesprächen die Glaubensgemeinschaft als Erzähl- und Tradierungsgemeinschaft erlebt bzw. aktualisiert werden kann. Das besondere Potenzial eines von der Tradition ausgehenden Erzählens liegt darin, dass die biblisch-christliche Tradition mehr als nur ein Antwort- und Deutungspotential für die Situationen, Probleme und Konflikte ist, die man auch ohne diese Tradition wahrnehmen, verstehen und bewältigen kann. Sie ist außerdem ein Erinnerungspotenzial, durch das (in manchmal überraschender Weise) überhaupt erst offen gelegt wird, in welcher Situation wir leben und welches unsere wirklichen Probleme und Konflikte sind. So gesehen lässt das Erzählen überlieferter Glaubensgeschichten in der Katechese manchmal überhaupt erst eine Situation entstehen, in der uns lebendiges Leben eröffnet wird und wir uns davon ergreifen lassen können.

Insgesamt haben das traditionsorientierte und das biografische Erzählen trotz ihrer unterschiedlichen Ansatzpunkte, Fragerichtungen und Potenziale in der Katechese das gemeinsame Anliegen, dem bipolaren Erschließungsprozess zwischen den Lebenserfahrungen heutiger Lernender und der biblisch-christlichen Tradition gerecht zu werden. In der Bipolarität zwischen den Inspirationen der Vergangenheit, die durch überlieferte Erzählungen kommuniziert werden, und den in biografischen Erzählungen heute lebender Menschen zum Ausdruck kommenden Lebenserfahrungen und Inspirationen stehen Katecheten vor der Herausforderung, eine Plattform der Begegnung zu gestalten. „Sie haben die Aufgabe, diese Plattform immer wieder wie ein Karussell anzustoßen und angestoßen sein zu lassen, sei es durch die Inspirationen der Vergangenheit, sei es durch die Inspirationen der Lebenden. Damit gibt es weder einen Traditionalismus, der die gegenwärtigen Erfahrungen verachtet, noch einen Fortschritts-Chauvinismus, der die vergangenen Erfahrungen missachtet" ${ }^{\text {"15 }}$.

\section{Die Rolle des Katecheten beim Erzählen}

Die Rolle des Katecheten, der diese Begegnungsplattform zwischen biblischchristlicher Überlieferung und aktuellen Lebenserfahrungen in Bewegung hält, hat verschiedene Facetten. Ein Katechet, der diese interaktive Plattform wie ein Karussell anstößt und angestoßen sein lässt, hat m.E. in erster Line die Rolle eines Moderators. Er ist als Moderator verantwortlich für die Gestaltung

${ }^{15}$ O. Fuchs, Verkündigung, in: Lexikon der Religionspädagogik, Bd. II, Neukirchen-Vluyn 2001, Verlag Neukirchener, S. 2177. 
des interaktiven Prozesses zwischen den Teilnehmenden (untereinander) und dem Gehalt der jeweiligen Erzählung. Zudem versucht er, eine geeignete Lernumgebung zu schaffen, in deren Rahmen die Teilnehmenden entdecken, wie ihre Lebenserfahrungen und die Erfahrungen von Glaubenden vor uns in einen konstruktiven Dialog kommen. Der moderierende Katechet nimmt die Teilnehmenden als aktive Subjekte wahr, die sich ihrerseits gleichberechtigt und aktiv in den Erzähl- und Gesprächsprozess der Katechese einbringen. In seiner Hauptrolle als Moderator des Erzählprozesses ist der Katechet zwar durchaus auch zuständig für die Auswahl geeigneter biographiebezogener Erzählimpulse einerseits und die kriteriengeleitete Auswahl exemplarischer überlieferter Erzählungen andererseits. Er trifft seine Auswahl aber in dem Bewusstsein, dass er selbst zwar durchaus ein Experte des Glaubens aber keinesfalls „im Besitz“ der Fülle des Glaubens der Kirche ist.

Wenn der Katechet sich in erster Line als Moderator des Erzähl- und Gesprächsprozesses versteht, kann das Erzählen in der Katechese nicht primär die Funktion der Informationsvermittlung haben, sondern es dient vor allem der Interaktion innerhalb der Katechesegruppe und dazu, die Plattform der Begegnung zwischen aktuellen und den überlieferten Erzählungen in Bewegung zu halten. Punktuell kann der moderierende Katechet dann durchaus auch eigene Glaubenserfahrungen als Impuls in das Gruppengespräch einbringen. In seiner Hauptrolle als Moderator müsste der Katechet jedoch auch sein Glaubenszeugnis gezielt als Dienst am Erzählprozess gleichberechtigter Erzähler und Zeugen einbringen, so dass sein Zeugnis nicht als die allein gültige Interpretation christlicher Wahrheit erscheint, sondern von der Katechesegruppe als Einladung zum Erzählen weiterer eigener Lebens- und Glaubenserfahrungen aufgenommen wird. Wenn der Katechet (die Erzählund Gesprächsprozesse) derart moderiert, kann es der Katechesegruppe gelingen, sich kritisch-konstruktiv über die Bedeutung eigener Erlebnisse zu verständigen und gemeinsame Glaubensvorstellungen zu konstruieren und $\mathrm{zu}$ profilieren. So trägt die Katechese zur Aktualisierung der Glaubenstradition bei und der Kirche als Erinnerungs- und Erzählgemeinschaft öffnet sich eine lebendige Zukunft. 


\section{Bibliography}

Emeis D., Schmitt K.H., Handbuch der Gemeindekatechese, Freiburg 1986, Verlag Herder.

Fuchs O., Bemerkungen und Beobachtungen zum Deutschen Katechetischen Kongress 1983, in: Deutscher Katecheten-Verein e.V. (Hrsg.), Erzähl mir deine Geschichte, Freiburg 1984, Verlag DKV, S. 152-154.

Fuchs O., Spuren auf meinem Glaubensweg, in: Deutscher Katecheten-Verein (Hrsg.), Dokumentation des Deutschen Katechetischen Kongresses 1983, Verlag Herder, S. 257-265.

Fuchs O., Verkündigung, in: Lexikon der Religionspädagogik, Bd. II, NeukirchenVluyn 2001, Verlag Neukirchener, S. 2170-2177.

Hasselhorn M., Gold A., Pädagogische Psychologie. Erfolgreiches Lehren und Lernen, Stuttgart 2006, Verlag Kohlhammer.

Karrer L., Grundvollzüge christlicher Praxis, in: H. Haslinger u.a. (Hrsg.), Handbuch Praktische Theologie, Bd. 2, Mainz 2000, Verlag Grünewald, S. 379-395.

Metz J. B., Kleine Apologie des Erzählens, in: Conc(D) 9 (1973), S. 336-341.

Niehl F.W., Erzählen, in: L. Rendle (Hrsg.), Ganzheitliche Methoden im Religionsunterricht, München 2007, Verlag Herder, S. 165-174.

Pottmeyer H.J., Die Suche nach der verbindlichen Tradition und die traditionalistische Versuchung der Kirche, in: D. Wiederkehr (Hrsg.), Wie geschieht Tradition? Überlieferung im Lebensprozess der Kirche (Quaestiones Disputatae 133), Freiburg 1991, Verlag Herder, S. 89-110.

Reiher D.,Praxis Christenlehre. Biblisches Erzählen inderSäkularität, in: G. Doyé, H. Kessler (Hrsg.), Konfessionslos und religiös. Gemeindepädagogische Perspektiven, Leipzig 2002, Verlag Leipziger, S. 145-158.

Schweitzer F., Elementarisierung - ein religionsdidaktischer Ansatz. Einführende Darstellung, in: ders., Elementarisierung im Religionsunterricht, NeukirchenVluyn 2003, Verlag Neukirchener, S. 9-30.

Schweitzer F., Elementarisierung nur der Inhalte - oder elementare Formen des Lernens?, in: ders., Elementarisierung im Religionsunterricht, NeukirchenVluyn 2003, Neukirchener, S. 187-201.

Weinert F. E., Lehren und lernen für die Zukunft. Ansprüche an das Lernen in der Schule, in: Pädagogische Nachrichten Rheinland-Pfalz (2/2000), S. 1-16.

Zimmermann S., Erzählen, in: G. Bitter u.a. (Hrsg.), Neues Handbuch religionspädagogischer Grundbegriffe, München 2002, Verlag Kösel, S. 481484. 